\title{
The Effects of Heat on Electronic Components
}

\author{
Adel Ahmed Almubarak
}

\begin{abstract}
Regardless of the cause of overheating, the general and primary effect of overheating on an electronic component is damage. In this paper, the effects of excessive levels heat on different types of electronic components are explored and discussed. Take note that included in this discussion are the causes of overheating to better understand and appreciate the overheating phenomenon as it applies to electronic components, as well as electronic assemblies and electronic devices. Through a review of literature, especially studies and engineering reference materials, this paper found out that at the micro-level, overheating causes material degradation on or in a specific electronic component. This degradation is further caused by cracks, expansion, and other structural deformation. Note that this degradation is also caused by different changes in the physical and chemical properties of a specific material due to exposure to high levels of heat. At macro-level, the effects of overheating center on systems failure due to degradation of different constituents and components, causeeffect loop, and neighboring-effect. Both micro-level and macro-level effects of overheating on electronic components increase the health and safety risk of an entire electronic assembly and electronic device. Hence, through a review of literature, this paper also discusses the techniques or processes for preventing overheating or reducing the negative effects of excessive levels of heat.
\end{abstract}

Keywords: overheating, electronic components, and thermal management

\section{INTRODUCTION}

Electronic components such as integrated circuits, transistors, diodes, resistors and capacitors, among others are designed and built to withstand particular amounts or levels of heat. Take note that when used in an electronic system such as an actual consumer electronic device, most of these electronic components generate heat. Examples are the diodes used in display technologies and integrated circuits in a central processing unit of a computer or mobile device. However, different internal and external situations can result in overheating that can potentially damage an electronic component. This research paper explores and discusses the effects of heat or more appropriately, of overheating of electronic components.

\section{Goals and Objectives}

The primary goal of this research paper is to determine the effects of overheating on electronic components. Aside from this, however, this paper also explores and discusses the different facets of overheating as far as electronic components are concerned. Thus, this paper also determines the causes of overheating and provides recommendations on how to protect electronic components from the damaging effects of excessive heat. These goals and objectives are achievable by referencing and reviewing related literatures.

\section{Causes of Overheating}

Overheating may be a result of direct and indirect, as well as internal and external influences. This means that there are different causes or factors at play why an electronic component becomes subjected to excessive levels of heat.

The study of B. Liao, A. A. Maznev, K. A. Nelson, and G. Chen (2016) mentioned the inevitable fact that consumer electronic devices such as portable laptops and smartphones are becoming more prone to overheating. This is because the physical dimensions of these devices are becoming smaller. To be specific, as demand for smaller devices becomes higher and more taxing, manufacturers of electronic components need to pack transistors into even small areas, and this cluttered engineering device increases overheating susceptibility due to reduced thermal flow. The study specifically demonstrated this phenomenon in a semiconductor. It revealed that as more electrons are packed into a small piece of a semiconductor such as a thin silicon wafer, the more electrons scatter phonons away. This scattering prevents the phonons from carrying away heat. Note that engineers typically recommend transistors and other electronic component to have a larger area and thicker physical dimension to increase thermal flow and reduce thermal boundary resistance (Korotkov, Samuelsen, \& Vasenco, 1994). Note that the required dimensional characteristics of electronic components and the actual devices or products in which they are used are indicative of design and engineering problem. In other words, design and engineering 
problem can be a direct and internal cause of overheating.

Other examples of bad design and engineering problems are faulty contact wiring and metaphysics coupling. As mentioned in the study of W. B. Yang, S. J. Mo, D. Lianga, \& F. J. Zhen (2014), faulty contact or contact wiring can result in overheating and sparks that can further generate fire. Furthermore, the study also said that multi-physics coupling engineering considerations, especially those that take into account the electric and temperature fields in the design of electronic components are inherently problematic. As a backgrounder, multiphysics coupling is a conventional engineering consideration in the design of miniaturized electronic component and production of compact form-factors. However, multi-physics coupling essentially increases the risks for internal problems of electronic components. This is because compact high power devices using miniaturized electronic components leads to higher power density (Dede, Lee, \& Nomura, 2014).

The article written and published by EE Publishers (2014) explained the different causes of overheating in capacitors. For instance, power dissipation induces heating in capacitors. However, excessive power dissipation results in the rise of temperature that can go beyond the capacity of transistors resulting in capacitor failure. Leakage current is another specific cause. A higher leakage current generates higher temperature across the capacitor due to loss of power. The article noted that aging generally causes higher leakage current because the oxide layer used in a capacitor dissolves over time. Surge voltages can also increase the internal temperature of a capacitor that can further lead to overheating.

Environmental factors can also lead to overheating. A reference material (Berk, 2009) lists down and discusses some of these factors. Fluctuating weather conditions or induced differences in ambient temperature can wear and tear electronic components. This is because temperature cycling can induce cracks over time as materials within a component expands and shrinks. An example of induced temperature cycling is constantly transporting a specific electronic component or an entire electronic device between two places with two different temperatures - such as using a smartphone or laptop outside where the temperature is warm and placing it inside an air-conditioned room. Humidity or the high moisture content of the surrounding air can also result in overheating because water vapor can contaminate electronic components and erode metal components or cause a short circuit. Of course, the hot temperature can promote internal overheating as well. Using a computer or a laptop in a hot environment will make internal heat distribution less efficient. Other causes of overheating are external bad contact and bad wiring (Yang, Mo, Lianga, \& Zhen, 2014) that can lead to excessive voltage surges and power dissipation (EE Publishers, 2014), improper use of an electronic device (Berk, 2009) such as using an incompatible auxiliary device as exemplified in using unrated cables and power adapters, overclocking of hardware components of computer devices, improper storage, and misuse and abuse, among others.

\section{Effects of Overheating}

Damage to an electronic component is the potential and direct effect of overheating. This is especially true if the component is unable to withstand the excessive levels of heat. Note that most, if not all, of failures in electrical and electronic components, are commonly associated with overheating and subsequent burning. This is why systems failure analysis of electronic components typically involves examining for evidences or indications of overheating and burning (Berk, 2009). The susceptibility an electronic assembly or electronic device to fail increases exponentially with temperature (Cengel \& Ghajar, 2015). In addition, there is a relationship between the performance, including the lifespan or lifecycle of an electronic component and its particular range of operating temperature (Mehoke, 2005). Temperature can essentially dictate the efficient performance of electronic components, as well as how long, will last. Several studies have uncovered and discussed how exactly overheating affects a particular electronic component. One dated study (Korotkov, Samuelsen, \& Vasenco, 1994) investigated the effects of overheating in a single-electron transistor or SET by directly and artificially applying excessive amounts of heat through current flow. The researchers demonstrated that the temperature of the central electrode of the SET remains finite because of the effects of electron tunneling. Note that electron tunneling is a phenomenon in which an electron moves through a potential energy barrier due to the accumulation of energy. In the research, the heat applied to the set resulted in an electronic tunneling that decreased the temperature difference between the central and outer electrodes. Further excessive heat resulted in hysteresis in the IV curve of the SET. As a backgrounder, in electronic circuitry, hysteresis is another phenomenon characterized by a change in the transfer characteristics of a circuit. While some hysteresis effects are intentional and needed in several electronic components, the researchers noted that the hysteresis they observed in the overheated SET displayed bi-stable characteristics.

Overheating also triggers damaging and cyclic interaction between particles. Another study (Liao, Maznev, Nelson, \& Chen, 2016) investigated 
the interaction between electrons and photons in a thin silicon wafer under the normal and excessive level of heats. To do this, the researchers used a three-pulse photo-acoustic spectroscopy. The investigation revealed that increasing the concentration of electrons in the silicon wafer resulted in electrons scattering the phonons. Such scattering prevented the phonons from carrying away heat. The findings essentially demonstrated a causeeffect loop of overheating. A small piece of silicon or any semiconductor packed with electrons would normally generate heat due to their small area. Moreover, the study revealed that more heat would be generated further because more electrons result in the scattering of phonons.

Several literatures, including reference materials, have also described the effects of overheating on specific types of electronic components. As an example, a detailed article written and published by EE Publishers (2014), a South African-based company that published a professional magazine about science and engineering, described and explained different specific effects of overheating in different types of capacitors. Ceramic capacitors, for example, can be subjected to excessive thermal stress and subsequent failure because high levels of heat result in dielectric breakdown. Overheating can also result in micro-cracks in a ceramic capacitor that will further result in moisture absorption and eventually, short circuit. The crack can further expand and degraded the material integrity of the capacitor. The damage due to overheating tends to be more pronounced in multilayer ceramic chip capacitors or MCCs. This is due to thermal coefficient of expansion of different ceramic materials within an MCC. In electrolytic capacitors, on the other hand, failure due to thermal stress is a result of excessive heat decomposing the electrolyte and the build up of gas that increases internal pressure. The damage can affect the entire printed circuit board. With regard to plastic film capacitors consisting of polystyrene, polyester, polycarbonate, and metalized polyester materials, among others, they are not prone to cracks due to overheating, unlike ceramic capacitors. Furthermore, in case of ruptures, they have the ability to self-heal. They are considerably more durable from heat and energy efficient than both ceramic capacitors and electrolytic capacitors. However, although prolonged exposure to excessive levels of heat will cause physical problems such as thermal expansion or structural deformation, such can result in combustion. This is especially true when film capacitors are used in alternating current circuits in which overheating can cause combustion failure. With regard to solid tantalum capacitors, overheating due to current surges can result in material disintegration and dormant damage to the dielectric over time. This is because solid tantalum capacitors, specifically the included titanium oxide films have surface imperfections and impurities that make it more vulnerable to thermal stress due to continuous exposure to excessive levels of heat. Take note that because a capacitor primarily functions as a device for storing electric charge and for filtering high-frequency components of the voltage, a damaged or degraded one will certainly affect the integrity of an entire circuit board. It is also important to mention that the quality and performance of capacitors can affect the entire power supply of an assembled component or circuitry. Usually, failures in capacitors lead to failures in other electronic components such as power transistors, metal-oxidesemiconductor field-effect transistor, and field-effect transistor, among others. Another example of an electronic component with specific descriptions of the effects of overheating is solid-state relay. The descriptions are found in a reference material (Platt \& Jansson, 2014) about electronic components. As a backgrounder, a sold-state relay is an electronic component that primarily functions as an electronic switching device. The switching mechanism happens with the application of a small voltage through its control terminals. The problem with this electronic component is that it is very sensitive to heat. Exposure to high levels of heat affects the rating for switching. In fact, as the temperature increases, the rating for switching diminishes. Overheating will cause the component not only to to fail due to material degradation and performance inefficiency but also in combustion. In assemblies with a crowded design, this burnout can affect nearby electronic components. Organic light-emitting diode or OLED is another example. Note that OLED is used as part of display technologies. A study (Yang, Wei, \& Zhang, 2012) that determines the transient thermal characterization of OLED mentioned that here is a linear relationship between optical performance and luminosity and junction temperature. In case of overheating, organic constituent of the OLED degrades. This means that more and prolonged exposure to excessive levels of heat increases the degradation level of OLED. This affects that luminosity of the diode. Apart from this, cracks can also form due to the thermal expansion coefficient of the different constituents of an OLED. The physical and chemical properties of specific constituents of an electronic component are also altered due to overheating. For instance, a study (Yun, Hongjin, \& Jianguo, 2011) determined the effects of overheating treatment on the microstructure of Terfenol-D alloy. As additional information, Terfenol-D alloy is a magnetostrictive material that demonstrates a strain upon exposure to a magnetic field. Emerging applications of this alloy include actuators, magnetomechanical sensors, and ultrasonic transducers, among others. The study revealed that 
exposing the alloy to different excessive levels of heat resulted in different microstructures and crystal orientation. It further revealed that overheating in general affected the pattern and mode of solidification of this alloy. The alterations in the physical property of Terfenol-D due to overheating will certainly be a problem, especially if this alloy is used as part of sensors and actuator devices. Another study (Yang, Mo, Lianga, \& Zhen, 2014) examined the specific miniscule effects of overheating on copper wires. Remember that copper is a common conductive material used as part of electronic components. Nonetheless, findings from this study revealed that overheating created a fatigue fracture surface on the copper wires. This means that excessive levels of heat can cause a directly noticeable physical effect on a particular material. In the case of conductive materials such as copper, a fatigue fracture can affect the physical properties and material integrity in several negative ways. The study noted that fatigue fracture surface observed at the overheated copper wire can further result in sparks that can generate combustion in the circuitry and subsequently, additional overheating and fire. Metals used for gluing or soldering together different components or specific constituents within a component can evaporate under high levels of heat. The result is a material breakdown of the component (EE Publishers, 2014). It has been noted that the thermal stress due to exposure to high levels of heat in the solder joints of a particular electronic component or an entire electronic assembly has been considered as one of the major causes of electronic systems failure (Cengel \& Ghajar, 2015). A reference material (Dede, Lee, \& Nomura, 2014) also mentioned that material coefficient of thermal expansion can result in structural deformation. This has been observed in specific constituents of an electronic component such as ceramics, metals, and plastics. Take note that thermal expansion is a phenomenon involving the expansion of a material in terms of size and volume upon exposure to specific levels of heat. Too much thermal expansion will certainly result in structural deformation. As mentioned, the structural deformation further results in thermally induced stress that can affect the integrity of a component. This problem is further aggravated if different materials within an electronic component suffer from thermal expansion and structural deformation due to exposure to higher levels of heat.

\section{SUMMARY AND CONCLUSION}

To reiterate the scope of this research paper, it is important to stress out the fact that heat is a normal byproduct of an electronic component or an entire electronic assembly that is under operation. This is the reason why electronic components are designed and built to withstand specific and certain levels of heat. However, excessive levels of heat results in overheating than in turn, result in damages to an electronic component.

Remember that from the discussion above, overheating may be a result of direct and indirect, as well as internal and external influences. Direct and internal influences usually pertain to the design and engineering considerations that make an electronic component or an entire electronic assembly prone to overheating. Compact electronic devices with large power requirement and miniaturized electronic components are susceptible to overheating because of the small area and limited dimension that makes heat transfer less efficient. Of course, poor design and engineering are also direct and internal influences. There is a high possibility that substandard electronic components or assemblies and devices might overheat due to lack of quality control and proper rating during their production. Aside from direct and internal influences, indirect and external influences can cause overheating. Examples of these are environmental weather conditions or ambient room temperature that can either promote overheating or lead to material degradation that will eventually result in overheating. High levels of humidity can cause overheating because of poor heat transfer and possible moisture contamination of electronic components. Other examples of indirect and external influences are improper use or misuse and abuse of electronic component, specifically of an electronic assembly and electronic device. Regardless of the cause of overheating, the general and primary effect of overheating on an electronic component is damage. This damage primarily affects the involved electronic component, but it can also affect other components in an assembly, thus resulting in overall system failure of the assembly or an electronic device. The aforementioned discussions provide a clear exploration and understanding of how exactly excessive levels of heat affect or damage different types of electronic component. One common but specific effect is material degradation. Electronic components such as ceramic capacitors and diodes are prone to cracks to overheating. After all, heat causes a material to expand. In a component composed of different constituents such as an OLED, cracks are also inevitable due to the different thermal coefficient of expansion of the materials. The heat essentially subjects different materials in different rate and size of expansion that on a collective level, result in disintegration.

Damaging and cyclic interaction between particles are also another effects of overheating. This phenomenon has been observed in minuscule semiconductors such as a thin silicon wafer. Concentration of electrons in the silicon wafer resulted in electrons scattering the phonons. Such 
scattering prevented the phonons from carrying away heat. Thus, under heat, the electrons packed in a semiconductor packed become excited. However, heat escape is possible because the packed and excited electrons result in the scattering of phonons. Phonons are essential in heat transfer. Overheating also affects the more specific constituents of an electronic component. Exposure to excessive levels of heat can lead to fatigue fracture surface of conductive materials such as copper. Overheating can also result in in different microstructures and crystal orientation of materials such as Terfanol-D alloy. Other effects of exposure to excessive levels of heat are evaporation of metals, or other materials use din soldering or in gluing together different parts of a particular electronic component or an electronic assembly. Nonetheless, these effects are indicative of the fact that overheating causes changes in the physical and chemical properties of specific constituents found in an electronic component. The secondary effects are material degradation of the actual constituents and subsequent failure of the electronic component. The changes in the physical and chemical properties affect the performance or in other words, the operation and function of an electronic component.

There are also other macro-scale effects of overheating. Certain types of capacitors, when exposed to high temperatures beyond their threshold capacity tend to burst and combust. This results in widespread damages in an electronic assembly. Combustion can result in fire obviously. An electronic device might explode and cause serious harm and danger. This phenomenon has been observed in capacitors and the different components of batteries. From the aforementioned effects of overheating of electronic components, it is safe to conclude that the most general and more troublesome effect is the dangers to end consumers. Apart from specific mechanical failure or systematic failures, an overheated electronic component, electronic assembly, or electronic device can cause serious injuries. A device, for instance, may explode and injure a user. It can also cause a fire. Because of this generalized effect, recommendations are given in the subsequent discussion. These recommendations explore the techniques or processes used to prevent overheating.

\section{Preventing Overheating}

There are technical and non-technical, as well as direct and indirect ways for preventing overheating. In the dated study of Korotkov, Samuelsen, \& Vasenco (1994) that involved investigating the effects of overheating in a SET, they recommended that one of the simplest ways to reduce heat in the central electrode of a SET is by increasing its area and thickness. Doing so would increase the heat flowing from electron gas to phonons. It would also reduce thermal boundary resistance, thus reducing the resistance of the central electrode to thermal flow and allowing it to become resistant to too much heat to a certain extent.

Another solution is the application of different enabling technologies for efficient cooling. This is especially true in components with multiphysics coupling systems. As mentioned by Dede, Lee, \& Nomura (2014), in evaluating the performance of an electronics systems that are designed after a multi-physics coupling engineering consideration, a combination of heat conduction, convection, and radiation should also be assessed and evaluated. They specifically recommended the use of conjugate or solid-fluid heat transfer that involves the use of a liquid substance that efficiently interacts with a heat sink to transport heat by conduction either through free or forced convection. The liquid substance is essentially the main transporter of heat as it travels through an electronic system. There are also other engineering and design considerations to prevent overheating. In capacitors, for example, a guideline (EE Publishers, 2014) mentioned that appropriate print circuit board layout techniques in which capacitors are placed away from other electronic components that tend to generate heat have been practiced by a lot of manufacturers. Integrating a heat-radiating protector between a capacitor and heat-prone electronic components is also a standard practice. Soldering should also be kept within the temperature profile of a specific type of capacitor. Note that this process creates heat, and excessive soldering can result in thermal stress and thermal damage.

Note that there are specific subfields and concepts in engineering that are designed or conceptualized to tackle overheating. Take note of thermal management as an example. A chapter (Mehoke, 2005) in the book about space systems introduced the use of thermal control subsystem in a spaceflight program. Accordingly, the goal of this subsystem is to control the temperature of all electronic components within a spacecraft during its entire operation. Such components include computers, instruments, and scientific sensors, among others. A standard practice in spacecraft technology is to ensure that an entire electronic assembly, electronic device, or equipment will not fail due to the overheating and subsequent failure of a specific electronic component. Another practice is to employ a failsafe mechanism in which operability and functionality will remain in case of electronic component failure. This is done by having a spare component or through the process of isolation. A reference material (Cengel \& Ghajar, 2015) about the fundamentals and application of heat and mass transfer outlines and discusses the different 
considerations in thermal management. Accordingly, one of the ways to ensure that a component will operate and function properly despite exposure to heat is the determination of thermal resistance. Another is the identification of thermal time constraint by the proper assembly and placement of a heat sink. A heat sink is commonly used and placed in electronic devices such as computers and display monitors. It functions as a specific device or a substance for absorbing and transferring excessive heat. Examples of heat sink are cooling fans found on the motherboard of a computer, liquid-cooling devices that are also used in computers, thermal interface materials such as thermal grease and thermal glue that increase thermal transfer efficiency, and aluminum-fined radiators and heat-spreaders, among others. Note that the thermal mass of heat sink can be regarded as a capacitor because it absolves and stores heat while subsequent thermal resistance is an electrical resistance. The relationship between this capacitor and electrical resistance forms a resistorcapacitor circuit that has a related time constant. This time constant can be used to accurately determine how well an entire assembly transfers heat from inside to outside. Another aspect of thermal management is the use of several cooling techniques. The material mentioned different types of cooling techniques to include conduction cooling, natural cooling using the principles of convection, cooling through the concept of radiation, cooling using forced-air, liquid cooling, and immersion cooling, among others. There are also manufacturing standards designed and implemented specifically to prevent overheating. In a book (Sangwine, 2007), manufacturers device and apply safety features specifically aimed at promoting electrical safety. Examples of these features are inaccessibility of live parts from end users and certain extent of proofing against mechanical abuse by ensuring durability and material integrity, among others. These manufacturers also subject a particular electronic component, an entire electronic assembly, or an electronic device under numerous stress tests to include exposure to different levels of temperature, drop test, vibration test, and impact test, among others. Note that this stress testing applies to prototypes only. Manufacturers have also employed industry-wide and government-enforced safety standards. These include putting appropriate markings on an electronic assembly or electronic device that indicate the nature of electrical supply, voltage rating, frequency of supply, and power consumption. Other manufacturers include additional safety information and warnings.

\section{REFERENCES}

[1] Berk, J. (2009). Systems Failure Analysis. Ohio: ASM International
[2] Cengel, Y. \& Ghajar, A. (2015). Heat and mass transfer: Fundamentals and applications (5th ed.). NY: McGraw-Hill

[3] Dede, E. M., Lee, J. \& Nomura, T. (2014). Multiphysics simulation: Electrochemical system applications and optimization. London: Springer-Verlag

[4] EE Publishers. (2014, July 8). Thermal stress on capacitors: Failure prevention. EE Publishers. Retrieved on 3 May 2017 from http://www.ee.co.za/article/thermal-stresscapacitors-failure-prevention.html

[5] Korotkov, A. N., Samuelsen, M. R., \& Vasenco, S. A. (1994). Effects of overheating in a single-electron transistor. Journal of Applied Physics. 76(6).10.1063/1.357424

[6] Liao, B., Maznev, A. A., Nelson, K. A., and Chen, G. (2016). Photo-excited charge carriers suppress sub-terahertz phonon mode in silicon at room temperature. Nature Communications. $7 . \quad$ DOI: 10.1038/ncomms 13174

[7] Mehoke, V. L. (2005). Spacecraft thermal control. In V. L. Pisacane (ed.), Fundamentals of Space Systems (2nd ed.). Oxford: Oxford University Press

[8] Platt, C. \& Jansson, F. (2014). "Solid-state relays." In Encyclopedia of Electronic Components Vol. 2: LEDs, LCDs, Audio, Thyristors, Digital Logic, and Amplification. CA: Maker Media, Inc.

[9] Sangwine, S. (2007). Electronic Components and Technology (3rd ed.). London: CRC Press

[10] Yang, L, Wei, B. \& Zhang, J. (2012). Transient thermal characterization of organic light-emitting diodes. Semiconductor Science and Technology. 27

[11] Yang, W. B., Mo, Lianga, S. J. \& Zhen, F. J. (2014). Numerical study of overheat fault in copper wire caused by bad contact base on multi-physics coupling. Paper presented at the 7th International Conference on Intelligent Computation Technology and Automation, Changsha, China. DOI: 10.1109/ICICTA.2014.103

[12] Yun, Y., Hongjin, W., \& Jianguo, L. (2011). The effect of overheating treatment on the microstructure of TbDyfe alloys. Paper presented at the International Conference on Advanced Technology Design and Manufacture, China. DOI: 10.1049/cp.2011.1055 